\title{
Evaluation OF NeUtrosophic SET APPROACH FILTERING TECHNIQUE FOR IMAGE DENOISING
}

\author{
J. Mohan ${ }^{1}$, A.P. Thilaga Shri Chandra ${ }^{2}$, Dr. V. Krishnaveni ${ }^{1}$ and \\ Dr. Yanhui Guo ${ }^{3}$ \\ ${ }^{1}$ Department of Electronics and Communication Engineering, P S G College of \\ Technology, Coimbatore, India. \\ jaimohan12@gmail.com, vk@ece.psgtech.ac.in \\ ${ }^{2}$ Department of Electronics and Communication Engineering, Sri Krishna College of \\ Engineering and Technology, Coimbatore, India. \\ thilagashrichandra@skcet.ac. in \\ ${ }^{3}$ Department of Radiology, University of Michigan, Ann Arbor, USA. \\ yanhuigemed.umich.edu
}

\begin{abstract}
This paper proposes an image denoising technique based on Neutrosophic Set approach of wiener filtering. A Neutrosophic Set (NS), a part of neutrosophy theory, studies the origin, nature, and scope of neutralities, as well as their interactions with different ideational spectra. Now, we apply the neutrosophic set into image domain and define some concepts and operators for image denoising. Here the image is transformed into NS domain, which is described using three membership sets: True (T), Indeterminacy $(I)$ and False $(F)$. The entropy of the neutrosophic set is defined and employed to evaluate the indeterminacy. The $\omega$-wiener filtering operation is used on $T$ and $F$ to decrease the set indeterminacy and remove noise. We have conducted experiments on a variety of noisy images using different types of noises with different levels. The performance of the proposed filter is compared with Median and Wiener filter based on Peak Signal to Noise Ratio (PSNR) and Root Mean Square Error (RMSE). The experimental results demonstrate that the proposed filter can remove noise automatically and effectively. Especially, it can process not only noisy images with different levels of noise, but also images with different kinds of noise well without knowing the type of the noise.
\end{abstract}

\section{KEYWORDS}

Image denoising, Neutrosophic Set, Wiener filtering, Entropy, PSNR, RMSE.

\section{INTRODUCTION}

An image is often corrupted by noise during its acquisition and transmission. Image denoising is used to remove the additive noise while retaining as much as possible the important signal features. Despite that a huge number of approaches has been proposed, denoise algorithms performance mainly depends on a suitable representation to describe the original image information and noise type.

The neutrosophic set approach had been applied into image processing such as image thresholding [1], image segmentation [2,3] and image denoising based on neutrosophic median filtering [4]. In this article, the new image denoising technique based on neutrosophic set approach of wiener filtering has been proposed. First the image is transformed into NS domain, which is described using three membership sets: True (T), Indeterminacy (I) and False (F). The

DOI : 10.5121/ijma.2012.4407 
The International Journal of Multimedia \& Its Applications (IJMA) Vol.4, No.4, August 2012

entropy of the neutrosophic set is defined and employed to evaluate the indeterminacy. The $\omega$ wiener filtering operation is used on $\mathrm{T}$ and $\mathrm{F}$ to decrease the set indeterminacy and remove noise. After the filtering process, the noise will be removed. The image will be transformed from NS domain into normal image. We have conducted experiments on a variety of noisy images using different types of noises with different levels. The experimental results demonstrate that the proposed approach can remove noise automatically and effectively. Especially, it can process not only noisy images with different levels of noise, but also images with different kinds of noise well without knowing the type of the noise.

The rest of paper is organized as follows. In section 2 we formulate the neutrosophic wiener filter for image denoising. The results for the proposed filtering method are discussed in section 3. Finally some conclusions are drawn in section 4.

\section{NeUTROSOPHIC IMAGE DENOISING}

Neutrosophy, a branch of philosophy introduced in [5] as a generalization of dialectics, studies the origin, nature and scope of neutralities, as well as their interactions with different ideational spectra. Neutrosophy theory considers proposition, theory, event, concept or entity, $<A>$ is in relation to its opposite $<$ Anti-A $>$ and the $<$ Neut-A $>$ which is neither $<A>$ nor $<$ Anti-A $>$. The neutrosophy is the basis of the neutrosophic logic, neutrosophic probability, and neutrosophic set and neutrosophic statistics [5]. In neutrosophic set, the indeterminacy is quantified explicitly and the truth-membership, indeterminacy-membership and falsity-membership are independent. The neutrosophic set is a general formal frame work which generalizes the concept of the classic set, fuzzy set [6], interval valued fuzzy set [7], intuitionistic fuzzy set [8], and interval valued intuitionistic fuzzy set [9], paraconsistent set, dialetheist set, paradoxist set and tautological set [5]. The definition of a neutrosophic set and its properties are described briefly.

\subsection{Neutrosophic Set}

Let $U$ be a Universe of discourse and a neutrosophic set $A$ is included in $U$. An element $x$ in set $A$ is noted as $x(T, I, F) . T, I, F$ are real standard and non standard sets of $]^{-} 0,1^{+}$[ with sup $T=t_{-}$sup, $\inf T=t_{-}$inf, sup $I=i_{-}$sup, inf $I=i_{-}$inf, $\sup F=f_{-}$sup, inf $F=f_{-}$inf and $n_{-}$sup $=t_{-}$sup + $i_{-} \sup +f_{-} \sup , n_{-} \inf =t_{-}$inf $+i_{-}$inf $+f_{-}$inf .

$\mathrm{T}, \mathrm{I}$ and $\mathrm{F}$ are called the neutrosophic components. The element $x(T, I, F)$ belongs to $A$ in the following way. It is $t \%$ true in the set, $i \%$ indeterminate in the set, and $f \%$ false in the set, where $t$ varies in $T, i$ varies in $I$ and $f$ varies in $F$ [5].

\subsection{Transform the Image into Neutrosophic Domain}

Let $U$ be a Universe of discourse and $W$ is a set of $U$, which is composed by bright pixels. A neutrosophic image $P_{N S}$ is characterized by three membership sets $T, I, F$. A pixel $P$ in the image is described as $P(T, I, F)$ and belongs to $W$ in the following way: It is $t$ true in the set, $i$ indeterminate in the set, and $f$ false in the set, where $t$ varies in $T, i$ varies in $I$ and $f$ varies in $F$. Then the pixel $P(i, j)$ in the image domain is transformed into the neutrosophic set domain $P_{N S}(i, j)=\{T(i, j), I(i, j), F(i, j)\}$, where $T(i, j), I(i, j)$ and $F(i, j)$ are the probabilities belong to white pixels set, indeterminate set and non white pixels set respectively [4], which are defined as: 
The International Journal of Multimedia \& Its Applications (IJMA) Vol.4, No.4, August 2012

$$
\begin{aligned}
& T(i, j)=\frac{\bar{g}(i, j)-\bar{g}_{\min }}{\bar{g}_{\max }-\bar{g}_{\text {min }}} \\
& \bar{g}(i, j)=\frac{1}{w \times w} \sum_{m=i-w / 2}^{i+w / 2} \sum_{n=j-w / 2}^{j+w / 2} g(m, n) \\
& I(i, j)=\frac{\delta(i, j)-\delta_{\min }}{\delta_{\max }-\delta_{\min }} \\
& \delta(i, j)=a b s(g(i, j)-\bar{g}(i, j)) \\
& F(i, j)=1-T(i, j)
\end{aligned}
$$

where $\bar{g}(i, j)$ is the local mean value of the pixels of the window. $\delta(i, j)$ is the absolute value of difference between intensity $g(i, j)$ and its local mean value $\bar{g}(i, j)$.

\subsection{Neutrosophic Image Entropy}

For a gray image, the entropy is utilized to evaluate the distribution of the gray levels. If the entropy is maximum, the intensities have equal probability. If the entropy is small, the intensity distribution is non-uniform.

Neutrosophic entropy of an image is defined as the summation of the entropies of three subsets $T, I$ and $F[4]$ :

$$
\begin{aligned}
& E n_{N S}=E n_{T}+E n_{I}+E n_{F} \\
& E n_{T}=-\sum_{i=\min \{T\}}^{\max \{T\}} p_{T}(i) \ln p_{T}(i) \\
& E n_{I}=-\sum_{i=\min \{I\}}^{\max \{I\}} p_{I}(i) \ln p_{I}(i) \\
& E n_{F}=-\sum_{i=\min \{F\}}^{\max \{F\}} p_{F}(i) \ln p_{F}(i)
\end{aligned}
$$

where $E n_{T}, E n_{I}$ and $E n_{F}$ are the entropies of sets $T, I$ and $F$ respectively. $p_{T}(i), p_{I}(i)$ and $p_{F}(i)$ are the probabilities of elements in $T, I$ and $F$ respectively, whose values equal to $i$.

\section{4. $\omega$-wiener Filtering Operation}

The values of $I(i, j)$ is employed to measure the indeterminate degree of element $P_{N S}(i, j)$. To make the set $I$ correlated with $T$ and $F$, the changes in $T$ and $F$ influence the distribution of element in $I$ and vary the entropy of $I$.

A $\omega$ - wiener filtering operation for $P_{N S}, \hat{P}_{N S}(\omega)$ is defined as:

$$
\hat{P}_{N S}(\omega)=P(\hat{T}(\omega), \hat{I}(\omega), \hat{F}(\omega))
$$


The International Journal of Multimedia \& Its Applications (IJMA) Vol.4, No.4, August 2012

$$
\begin{aligned}
& \hat{T}(\omega)=\left\{\begin{array}{cc}
T & I<\omega \\
\hat{T}_{\omega} & I \geq \omega
\end{array}\right. \\
& \hat{T}_{\omega}(i, j)=\underset{(m, n) \in S_{i, j}}{\text { wiener }}\{T(m, n)\} \\
& \hat{F}(\omega)= \begin{cases}F & I<\omega \\
\hat{F}_{\omega} & I \geq \omega\end{cases} \\
& \hat{F}_{\omega}(i, j)=\underset{(m, n) \in S_{i, j}}{\text { wiener }\{F(m, n)\}} \\
& \hat{I}_{\omega}(i, j)=\frac{\delta_{\hat{T}}(i, j)-\delta_{\hat{T} \min }}{\delta_{\hat{T} \max }-\delta_{\hat{T} \min }} \\
& \delta_{\hat{T}}(i, j)=a b s(\hat{T}(i, j)-\overline{\hat{T}}(i, j)) \\
& \overline{\hat{T}}(i, j)=\frac{1}{w \times w} \sum_{m=i-w / 2}^{i+w / 2} \sum_{n=j-w / 2}^{j+w / 2} \hat{T}(m, n)
\end{aligned}
$$

where $\delta_{\hat{T}}(i, j)$ is the absolute value of difference between intensity $\hat{T}(i, j)$ and its local mean value $\overline{\hat{T}}(i, j)$ at $(i, j)$ after $\omega$ - wiener filtering operation.

The summary of image denoising method based on neutrosophic set approach of wiener filtering is described as below (see Figure 1):

Step 1: Transform the image into NS domain;

Step 2: Use $\omega$ - wiener filtering operation on the true subset $T$ to obtain $T_{\omega}$;

Step 3: Compute the entropy of the indeterminate subset $\hat{I}_{\omega}, E n_{\hat{I}_{\omega}}(i)$;

Step 4: if $\frac{E n_{\hat{I}_{\omega}}(i+1)-E n_{\hat{I}_{\omega}}(i)}{E n_{\hat{I}_{\omega}}(i)}<\delta$, go to Step 5; Else $T=\hat{T}_{\omega}$, go to Step 2;

Step 5: Transform subset $\hat{T}_{\omega}$ from the neutrosophic domain into the gray level domain.

\section{RESULTS AND DISCUSSION}

In the experiments, Lena and Cameraman images are used to evaluate the performance of the proposed method and compared with two filters such as median filter and wiener filter. Different kinds of noise such as Gaussian noise and salt and pepper noise with different levels are added into the image.

The performance of the denoising algorithm is measured by using Peak signal to Noise Ratio (PSNR) and the Root Mean Squared Error (RMSE). PSNR in decibel (dB) is computed using the following formula:

$$
P S N R=-10 \log \left[\frac{\sum_{i=0}^{i=H-1} \sum_{j=0}^{j=W-1}\left(I(i, j)-I_{d}(i, j)\right)^{2}}{M \times N \times 255^{2}}\right]
$$

RMSE is the objective quality measure that quantifies the deviation of estimated values from the true value. The RMSE is measured as: 
The International Journal of Multimedia \& Its Applications (IJMA) Vol.4, No.4, August 2012

$$
R M S E=\sqrt{\frac{\sum_{i} \sum_{j}\left[I(i, j)-I_{d}(i, j)\right]^{2}}{M \times N}}
$$

where $I(i, j)$ and $I_{d}(i, j)$ represent the intensities of pixels $(i, j)$ in the original image and denoised image respectively, $M \times N$ is the size of the image.

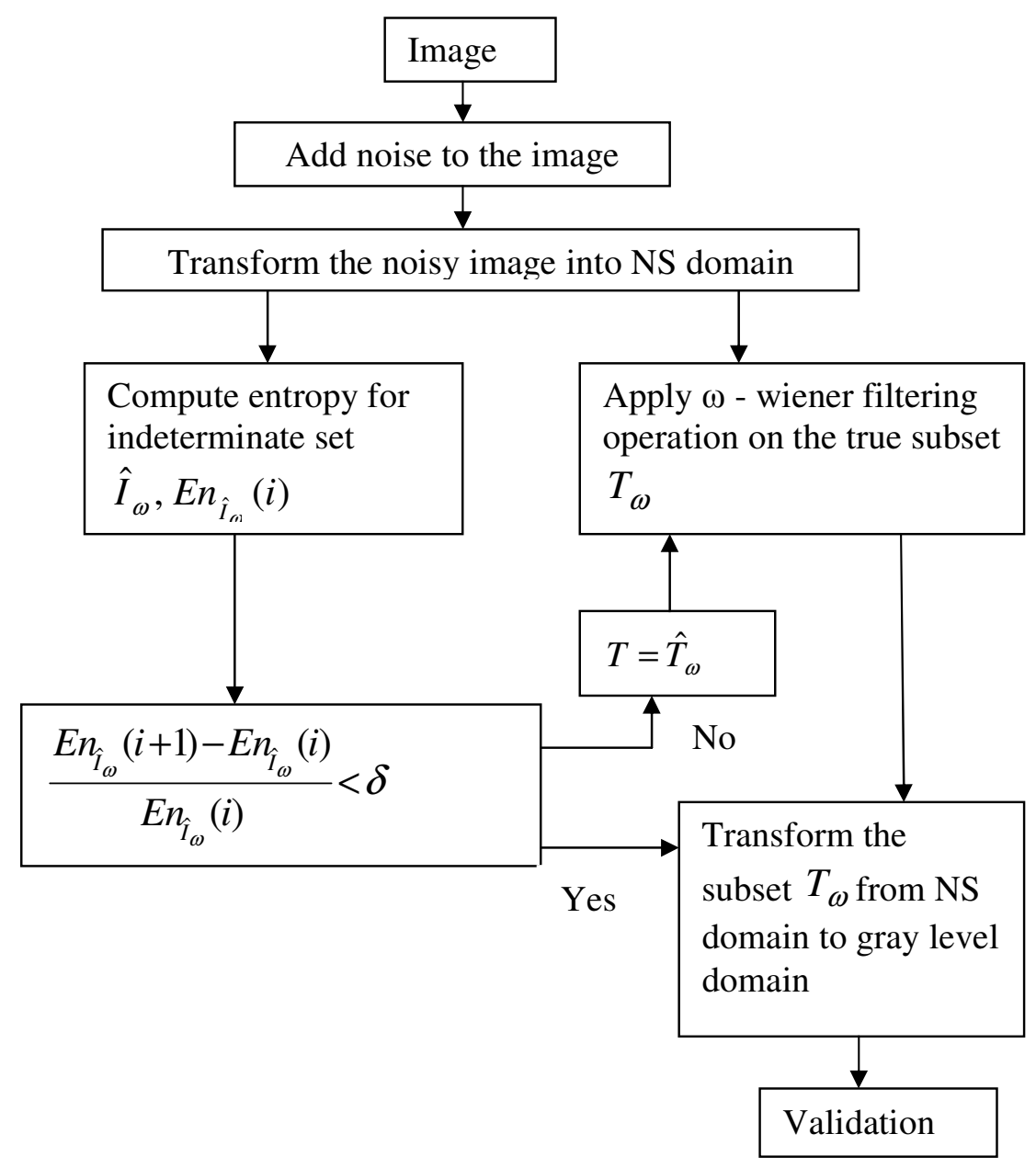

Figure 1. Image Denoising: Neutrosophic Set approach of wiener filtering

First, the proposed denoising algorithm is compared with median and wiener filter in removing Gaussian noise with different noise levels added to the Lena and cameraman images. From the comparison on visual and quantity measures in Figure 2, conclude that the Neutrosophic approach of wiener filtering is not only removes the Gaussian noise and also achieves the low RMSE. The comparison of these filters based on RMSE with different noise levels is shown in Figure 3a for Lena image and Figure $3 \mathrm{~b}$ for Cameraman image.

Second, the proposed filter is employed to remove the other type of noise such as salt and pepper noise. From Figure 4, the proposed filter outperforms the other two filters. The comparison of RMSE values of different filters are shown in Figure 5a for Lena image and Figure 5b for Cameraman image. The PSNR values of the proposed filter, median and wiener filter for Lena and Cameraman images with Gaussian and Salt and pepper noise is tabulated in Table 1 and Table 2. 


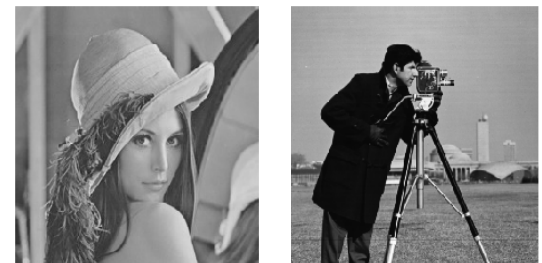

(a)

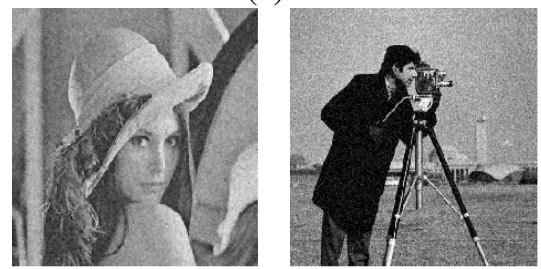

(c)

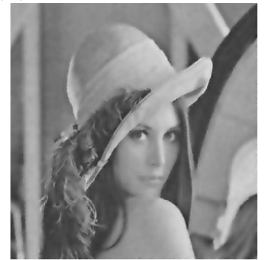

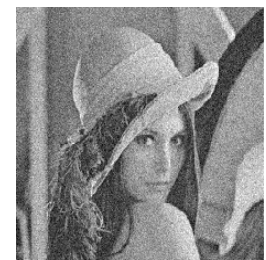

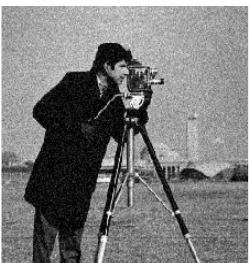

(b)

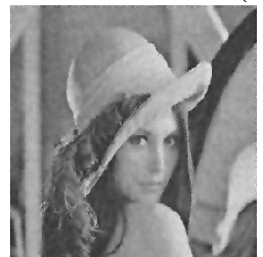

(d)

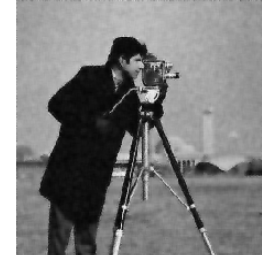

(e)

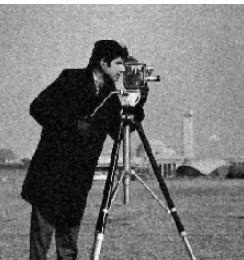

Figure 2.(a) Original Image, (b) Noisy image (Gaussian noise): Lena (PSNR 29.57), Cameraman (PSNR 29.82) (c) Denoised using Median Filter: Lena (PSNR 31.03), Cameraman (PSNR 31.53)

(d) Denoised using Wiener: Lena (PSNR 31.4), Cameraman (PSNR 33.01) $\quad$ (e) Denoised using NS Wiener: Lena (PSNR 33.63), Cameraman (PSNR 33.59)

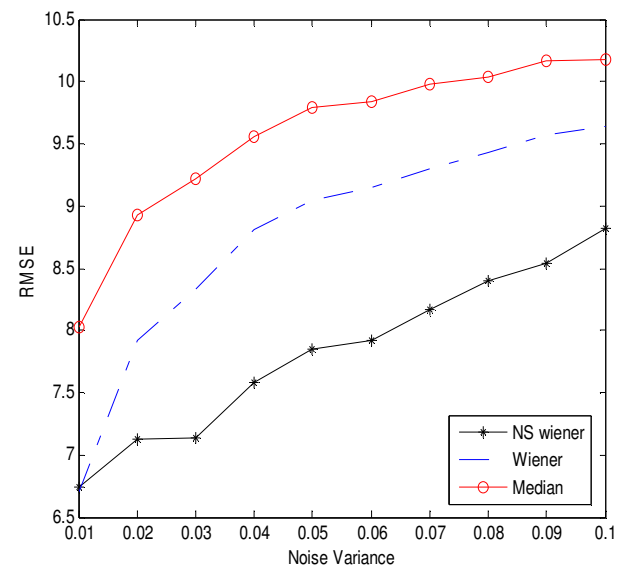

(a)

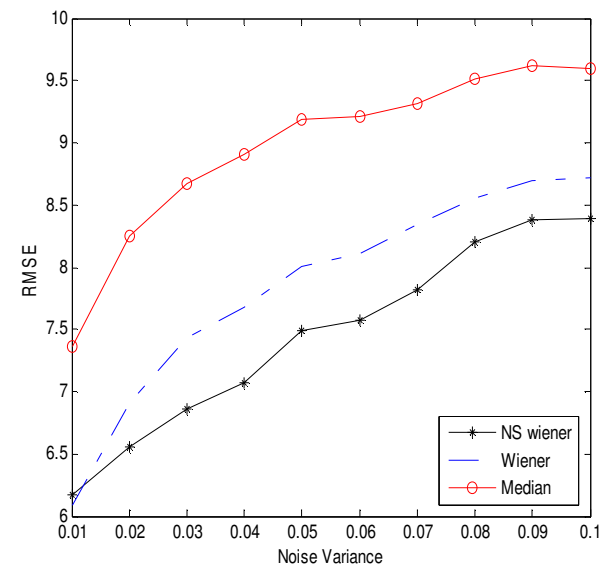

(b)

Figure 3. Comparison of Median, Wiener, and NS Wiener Filters for Gaussian noise based on the RMSE values: (a) Lena (b) Cameraman 
The International Journal of Multimedia \& Its Applications (IJMA) Vol.4, No.4, August 2012

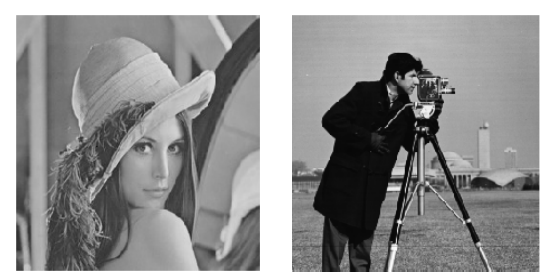

(a)

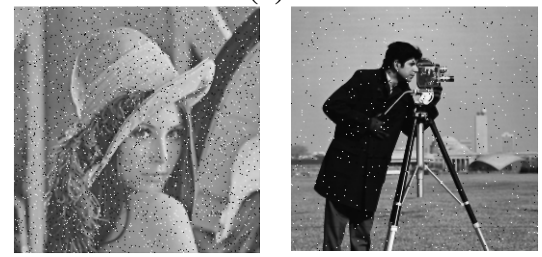

(c)

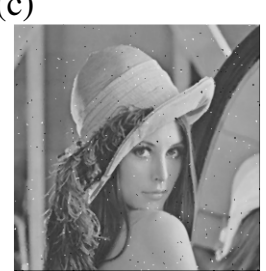

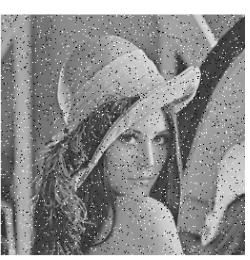

(b)
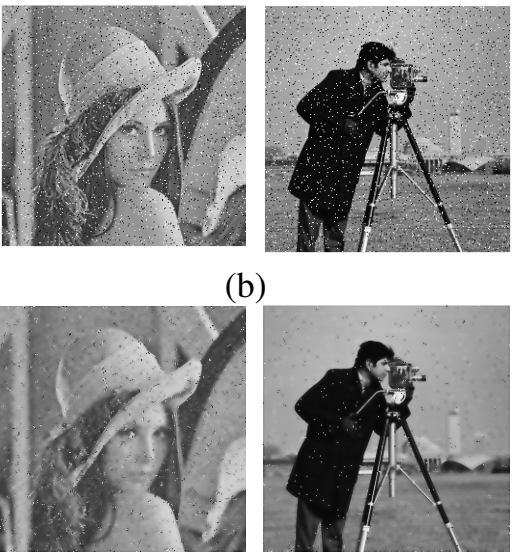

(d)

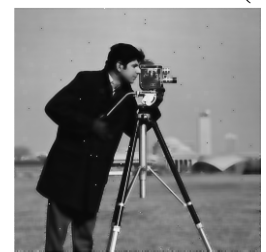

(e)

Figure 4. (a) Original Image, (b) Noisy image (salt and pepper noise): Lena (PSNR 30.28), Cameraman (PSNR 30.32) (c) Denoised using Median Filter: Lena (PSNR 32.24), Cameraman (PSNR 32.07) (d) Denoised using Wiener: Lena (PSNR 35.28), Cameraman (PSNR 33.93)

Denoised using NS Wiener: Lena (PSNR 36.11), Cameraman (PSNR 35.61)

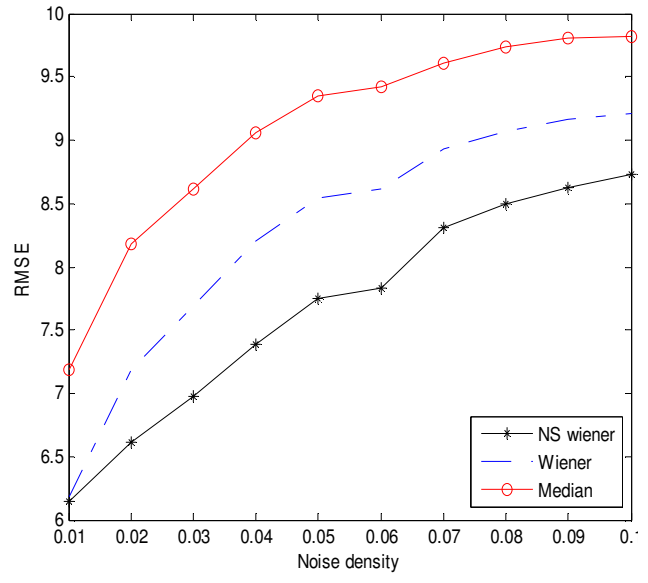

(a)

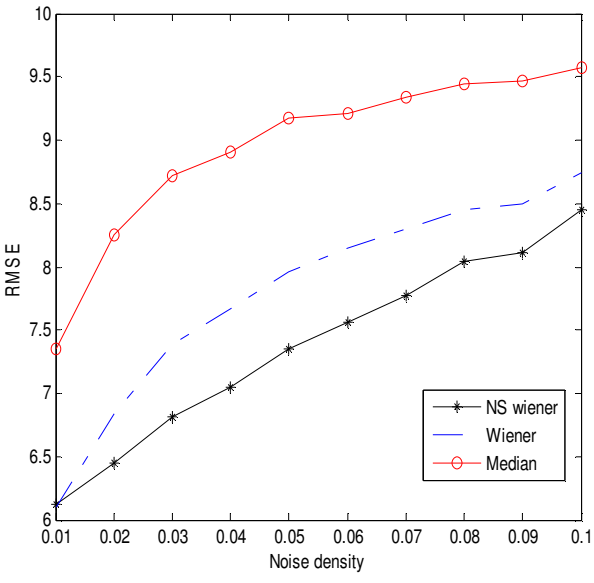

(b)

Figure 5. Comparison of Median, Wiener, and NS Wiener Filters for salt and pepper noised based on the RMSE values: (a) Lena (b) Cameraman. 
The International Journal of Multimedia \& Its Applications (IJMA) Vol.4, No.4, August 2012

Table 1 Comparison of Median, Wiener, NS Wiener Filters Based on PSNR in dB for Gaussian Noise

\begin{tabular}{|c|c|c|c|c|c|c|}
\hline \multirow{3}{*}{ 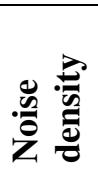 } & \multicolumn{6}{|c|}{ PSNR (dB) } \\
\hline & \multicolumn{2}{|c|}{ Median } & \multicolumn{2}{|c|}{ Wiener } & \multicolumn{2}{|c|}{ NS wiener } \\
\hline & Lena & $\begin{array}{l}\text { Camera } \\
\text { man }\end{array}$ & Lena & $\begin{array}{l}\text { Camera } \\
\text { man }\end{array}$ & Lena & $\begin{array}{l}\text { Camera } \\
\text { man }\end{array}$ \\
\hline 0.001 & 31.97 & 31.96 & 35.29 & 34.51 & 35.24 & 34.51 \\
\hline 0.002 & 31.79 & 31.81 & 34.49 & 33.85 & 35.28 & 34.32 \\
\hline 0.003 & 31.68 & 31.7 & 33.8 & 33.29 & 35.08 & 34.13 \\
\hline 0.004 & 31.51 & 31.61 & 33.2 & 32.92 & 34.87 & 34.06 \\
\hline 0.005 & 31.39 & 31.48 & 32.74 & 32.55 & 34.65 & 33.95 \\
\hline 0.006 & 31.32 & 31.43 & 32.39 & 32.31 & 34.51 & 33.89 \\
\hline 0.007 & 31.29 & 31.37 & 32.09 & 31.89 & 34.46 & 33.74 \\
\hline 0.008 & 31.12 & 31.32 & 31.74 & 31.78 & 34.22 & 33.78 \\
\hline 0.009 & 31.09 & 31.21 & 31.51 & 31.46 & 34.09 & 33.56 \\
\hline
\end{tabular}

Table 2. Comparison of Median, Wiener, NS Wiener Filters Based on PSNR in dB for Salt and Pepper noise

\begin{tabular}{|c|c|c|c|c|c|c|}
\hline \multirow{3}{*}{ 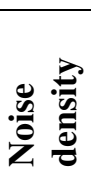 } & \multicolumn{6}{|c|}{ PSNR (dB) } \\
\hline & \multicolumn{2}{|c|}{ Median } & \multicolumn{2}{|c|}{ Wiener } & \multicolumn{2}{|c|}{ NS wiener } \\
\hline & Lena & $\begin{array}{l}\text { Camera } \\
\text { man }\end{array}$ & Lena & $\begin{array}{l}\text { Camera } \\
\text { man }\end{array}$ & Lena & $\begin{array}{l}\text { Camera } \\
\text { man }\end{array}$ \\
\hline 0.01 & 32.29 & 32.06 & 36.16 & 33.81 & $\mathbf{3 7 . 8 8}$ & 35.66 \\
\hline 0.02 & 32.02 & 31.82 & 35.74 & 33.46 & 36.73 & 35.38 \\
\hline 0.03 & 31.78 & 31.57 & 35.23 & 32.94 & 36.04 & 35.03 \\
\hline 0.04 & 31.52 & 31.31 & 34.94 & 32.56 & 35.66 & 34.81 \\
\hline 0.05 & 31.28 & 31.06 & 34.53 & 32.21 & 35.31 & 34.55 \\
\hline 0.06 & 30.99 & 30.8 & 34.15 & 31.82 & 34.99 & 34.32 \\
\hline 0.07 & 30.72 & 30.56 & 33.79 & 31.52 & 34.71 & 34.03 \\
\hline 0.08 & 30.51 & 30.32 & 33.44 & 31.27 & 34.41 & 33.88 \\
\hline 0.09 & 30.25 & 30.04 & 33.27 & 30.81 & 34.21 & 33.61 \\
\hline
\end{tabular}

\section{Conclusions}

In this paper, a novel image denoising technique based on neutrosophic set approach of wiener filtering has been proposed. The image is described as a NS set using three membership sets $T, I$ and $F$. The entropy in neutrosophic image domain is defined and employed to evaluate the indetermination. The wiener filter is applied to reduce the set's indetermination and remove the noise in the image. We have conducted experiments on a variety of noisy images using different types of noises with different levels. The performance of the proposed filter is compared with Median and Wiener filter based on Peak Signal to Noise Ratio (PSNR) and Root Mean Square Error (RMSE). The experimental results demonstrate that the proposed approach can remove noise automatically and effectively. Especially, it can process not only noisy images with different levels of noise, but also images with different kinds of noise well without knowing the type of the noise. The properties of neutrosophic image will achieve more applications in processing and computer vision. 
The International Journal of Multimedia \& Its Applications (IJMA) Vol.4, No.4, August 2012

\section{REFERENCES}

[1] H. D. Cheng and Y. Guo, "A new neutrosophic approach to image thresholding,"New Mathematics and Natural Computation, vol. 4, no. 3, pp.291-308, 2008.

[2] Yanhui Guo and H. D. Cheng, "New Neutrosophic approach to image segmentation," Pattern Recognition, vol. 42, no. 5, pp. 587-595, May, 2009.

[3] M. Zhang, L. Zhang, H. D. Cheng, "A Neutrosophic approach to image segmentation based on Watershed method," Signal Processing, vol. 90, no. 5, pp.1510-1517, May 2010.

[4] Yanhui Guo, H. D. Cheng and Y. Zhang, "A New Neutrosophic approach to Image Denoising," New Mathemetics and Natural Computation, vol. 5, no. 3, pp. 653-662, 2009.

[5] F. Samarandache, A unifying field in logics Neutrosophic logic, in Neutrosophy. Neutrosophic Set, Neutrosophic Probability, $3^{\text {rd }}$ edition, American Research Press, 2003.

[6] L. A. Zadeh, "Fuzzy sets," Inform and control, vol. 8, pp. 338-353, 1965.

[7] I. Turksen, "Interval valued fuzzy sets based on normal forms," Fuzzy Sets and Systems, vol. 20, pp. 191-210, 1986.

[8] K. Atanassov, "Intuitionistic fuzzy sets," Fuzzy sets and systems, vol. 20, pp. 87-96, 1986.

[9] K. Atanassov, "More on Intuitionistic fuzzy sets," Fuzzy sets and systems, vol. 33, pp. 37-46, 1989.

Authors

J. Mohan received the B.E (ECE) degree from Bharathidasan University, Trichy and M.E degree from Sathyabama University, Chennai. Currently précising his $\mathrm{PhD}$ in Medical image processing in PSG College of Technology, Anna University Chennai. Presently working at P.A. college of Engineering and Technology with an experience of 11 years in the teaching field.

A. P. Thilaga Shri Chandra received the B.E (ECE) degree in 2003 from Anna University, Chennai, and M.E (Communication Systems) degree in 2007 from Anna University, Chennai. Presently working as an Assistant Professor in Sri Krishna College of Engineering and Technology, Coimbatore.

Dr. V. Krishnaveni is Associate Professor in the Department of Electronics and Communication Engineering, PSG College of Technology, Coimbatore. She did her BE (ECE) and ME (Communication Systems) from Bharathiar University, Coimbatore and $\mathrm{PhD}$ in the area of Biomedical Signal Processing from Anna University Chennai. She has more than 15 years of teaching experience at UG and PG levels. She has published more than 25 papers in various international/ national journals/conferences. She is a reviewer for more than 5 international journals which includes IEEE Transactions on Biomedical Engineering. Her research interests include digital signal processing and image processing with applications to biomedical signals and images.

Dr. Yanhui Guo received B. S. degree in Automatic Control from Zhengzhou University, P. R. China, in 1999, the M.S. degree in Pattern Recognition and Intelligence System from Harbin Institute of Technology, Harbin, Heilongjiang Province, P. R. China, in 2002, and the Ph.D. degree in the Department of Computer Science, Utah State University, USA, in 2010. He is currently working in the Department of Radiology, University of Michigan. His research interests include image processing, pattern recognition, medical image processing, computer-aided
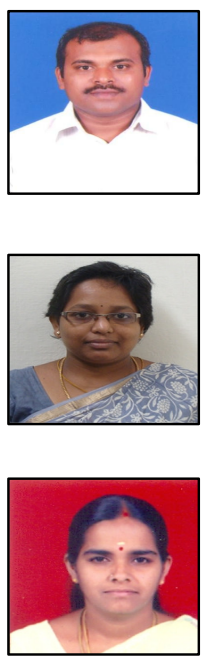
detection/diagnosis, fuzzy logic, and neutrosophic theory.

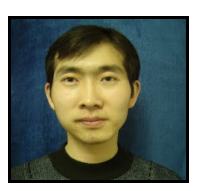

